\title{
Implicative Almost Distributive Lattices
}

\author{
Berhanu Assaye Alaba, Mihret Alamneh and Tilahun Mekonnen
}

\begin{abstract}
In this paper, we introduce the concept of Implicative Almost Distributive Lattices (IADLs) as a generalization of implicative algebra in the class of Almost Distributive Lattices. We discuss some properties of IADL and derive some equivalent conditions in IADLs. We also discuss some characterizations of IADL to become an implicative algebra.
\end{abstract}

Index Terms-Almost distributive lattices, implicative algebra.

\section{INTRODUCTION}

$\mathbf{T}$ O establish an alternative logic for knowledge representation and reasoning, $\mathrm{Xu}$ [1] proposed a logical algebralattice implication algebra in 1993 by combining algebraic lattice and implication algebra. Lattice implication algebra is an important non-classical logical algebra, it has been studied by researchers. Lattice-valued logic is an important form of many-valued logic which extends the field of truth-values to lattices. More important, lattice-valued logic can represent the uncertainty, especially the incomparable property of people's thinking, judging and decision. In lattice implication algebra, the lattice is defined to describe uncertainties, especially for the incomparability and the implication is designed to describe the ways of human's reasoning. Xu et al. [2] have established the lattice valued propositional logic $L p(X)$ and lattice valued first ordered logic $\operatorname{Lf}(\mathrm{X})$ and the gradual lattice valued propositional logic Lvpl and the gradual lattice valued first order logic Lvfl [3] by taking lattice implication algebra as a truth value field. Venkateswarlu Kulluru and Berhanu Bekele [4] introduced the concept of implicative algebras and obtained certain properties. Further they proved that implicative algebra is equipped with a structure of a bounded lattice and proved that it is a lattice implication algebra [4]. The concept of an Almost Distributive Lattice (ADL) was introduced in 1981 by U.M. Swamy and G.C.Rao [5] as a common abstraction to most of the existing ring theoretic and lattice theoretic generalization of Boolean algebra. In this paper, we introduce the concept of an Implicative Almost Distributive Lattice (IADL) as a generalization of an implicative algebra in the class of ADLs. We also characterize an IADL in terms of the set of all of its principal ideals. We prove some properties and equivalence condition in an implicative almost distributive lattice and also characterize that Implicative Almost Distributive lattice becomes an Implicative Algebra.

In the following, we give some important definitions and results that will be useful in the study of IADLs.

Manuscript received October 12, 2017; accepted November 29, 2017.

The authors are with the Department of Mathematics, College of Science, Bahir Dar University, Bahir Dar, Ethiopia. E-mail: berhanu_assaye@yahoo.com, mihretmahlet@yahoo.com, tilametekek@yahoo.com

\section{Preliminaries}

Definition 2.1 ([5]): An algebra $(L, \vee, \wedge, 0)$ of type $(2,2,0)$ is called an Almost Distributive Lattice (ADL) with 0 if it satisfies the following axioms:

1) $(x \vee y) \wedge z=(x \wedge z) \vee(y \wedge z)$

2) $x \wedge(y \vee z)=(x \wedge y) \vee(x \wedge z)$

3) $(x \vee y) \wedge y=y$

4) $(x \vee y) \wedge x=x$

5) $x \vee(x \wedge y)=x$

6) $0 \wedge x=0$, for all $x, y, z \in L$

Definition 2.2 ([5]): Let L be a non empty set. Fix $x_{0} \in L$. For any $x, y \in L$, define $x \vee y=x, x \wedge y=y$ if $x \neq x_{0} ; x_{0} \wedge y=x_{0}$ and $x_{0} \vee y=y$.Then $\left(L, \vee, \wedge, x_{0}\right)$ is called a discrete ADL with $x_{0}$ as its 0 .

If $(L, \vee, \wedge, 0)$ is an ADL, for any $x, y \in L$, define $x \leq y$ if and only if $x=x \wedge y$ or equivalently $x \vee y=y$, then $\leq$ is a partial ordering on $\mathrm{L}$.

Theorem 2.3 ([5]): Let $(L, \vee, \wedge, 0)$ be an ADL. Then for any $x, y, z \in L$, we have the following:

1) $x \vee y=x \Leftrightarrow x \wedge y=y$

2) $x \vee y=y \Leftrightarrow x \wedge y=x$

3) $x \wedge y=y \wedge x=x$ whenever $x \leq y$

4) $\wedge$ is associative

5) $x \wedge y \wedge z=y \wedge x \wedge z$

6) $(x \vee y) \wedge z=(y \vee x) \wedge z$

7) $x \wedge y \leq y$ and $x \leq x \vee y$

8) If $x \leq z$ and $y \leq z$, then $x \wedge y=y \wedge x$ and $x \vee y=y \vee x$

Theorem 2.4 ([5]): In an ADL L, the following are equivalent:

1) $\mathrm{L}$ is a distributive lattice

2) The poset $(L, \leq)$ is directed above

3) $x \vee y=y \vee x$ for all $x, y \in L$

4) $x \wedge y=y \wedge x$ for all $x, y \in L$

5) $(x \wedge y) \vee z=(x \vee z) \wedge(y \vee z)$ for all $x, y, z \in L$

6) $\theta=\{(x, y) \in L \times L: y \wedge x=x\}$ is anti symmetric

Definition 2.5: In an ADL L, $(L, \leq)$ be a poset, $S \subseteq L$ and $a \in L$.Then

1) a is celled a lower bound of $\mathrm{S}$ if $a \leq x$ for all $x \in S$.

2) a is an upper bound of $\mathrm{S}$ if $x \leq a$ for all $x \in S$.

3) $\mathrm{a}$ is called the greatest lower bound or g.l.b or infimum of $\mathrm{S}$ if $\mathrm{a}$ is a lower bound of $\mathrm{S}$ and for any lower bound $\mathrm{b}$ of $\mathrm{S}$, we have $b \leq a$.In this case we write $\mathrm{a}=$ g.l.b $\mathrm{S}$ or $\mathrm{a}=\inf \mathrm{S}$.

4) a is called the least upper bound or l.u.b or supremum of $\mathrm{S}$ if $\mathrm{a}$ is an upper bound of $\mathrm{S}$ and for any upper bound $\mathrm{b}$ of $\mathrm{S}$, we have $a \leq b$. In this case we write a $=1$.u.b $\mathrm{S}$ or $\mathrm{a}=\sup \mathrm{S}$.

Definition 2.6 ([5]): An element $\mathrm{m}$ in ADL $\mathrm{L}$ is called maximal if for any $x \in L, m \leq x$ implies $\mathrm{m}=\mathrm{x}$. 
Proposition 2.7 ([5]): If an ADL L has maximal elements, then for each $x \in L$ there exists a maximal element $\mathrm{m}$ such that $x \leq m$.

The following definitions, lemma and theorems are in an Implicative Algebra that is useful in our study.

Definition 2.8 ([4]): An algebra $(L, \rightarrow, /, 0,1)$ of type $(2,1,0,0)$ is called implicative algebra if it satisfies the following conditions:

1) $x \rightarrow(y \rightarrow z)=y \rightarrow(x \rightarrow z)$

2) $1 \rightarrow x=x$

3) $x \rightarrow 1=1$

4) $x \rightarrow y=y^{\prime} \rightarrow x^{\prime}$

5) $(x \rightarrow y) \rightarrow y=(y \rightarrow x) \rightarrow x$

6) $0^{\prime}=1$, for $x, y, z \in L$

Definition 2.9 ([4]): A relation $\leq$ on an implicative algebra $\mathrm{L}$ is defined as follows: $x \leq y \Leftrightarrow x \rightarrow y=1$ for all $x, y \in L$.

Lemma 2.10 ([4]): In an implicative algebra L, the following conditions hold:

1) $x \rightarrow x=1$

2) $1^{\prime}=0$

3) $0 \rightarrow x=1$

4) $x \rightarrow y=1=y \rightarrow x \Leftrightarrow x=y$

5) $x \rightarrow y=1$ and $y \rightarrow z=1$, then $x \rightarrow z=1$

6) $x \leq y \Leftrightarrow z \rightarrow x \leq z \rightarrow y$ and $y \rightarrow z \leq x \rightarrow z$

7) $((x \rightarrow y) \rightarrow y) \rightarrow y=x \rightarrow y$

8) $(x \rightarrow y) \rightarrow[(y \rightarrow z) \rightarrow(x \rightarrow z)]=1$

9) $\left(x^{\prime}\right)^{\prime}=x$

10) $x^{\prime}=x \rightarrow 0$, all $x, y, z \in L$.

We define two binary operations $\vee$ and $\wedge$ on an implication algebra by

$x \vee y=(x \rightarrow y) \rightarrow y=(y \rightarrow x) \rightarrow x$,

$x \wedge y=\left[(x \rightarrow y) \rightarrow x^{\prime}\right]^{\prime}=\left[(y \rightarrow x) \rightarrow y^{\prime}\right]^{\prime}$ for all $x, y \in L$

Theorem 2.11 ([4]): In an implicative algebra L, the following conditions hold:

1) $x \wedge y \leq x, y \leq x \vee y$

2) $x \leq y, x \leq z$ implies $x \leq y \wedge z$

3) $y \leq x, z \leq x$ implies $(y \vee z) \leq x$

4) $(x \vee y) \rightarrow z \leq x \rightarrow z$ and $(x \vee y) \rightarrow z \leq y \rightarrow z$

5) $x \rightarrow z \leq(x \wedge y) \rightarrow z$ and $y \rightarrow z \leq(x \wedge y) \rightarrow z$

6) $(x \vee y) \rightarrow z=(x \rightarrow z) \wedge(y \rightarrow z)$

7) $(x \wedge y) \rightarrow z=(x \rightarrow z) \vee(y \rightarrow z)$

8) $x \rightarrow(y \wedge z)=(x \rightarrow y) \wedge(x \rightarrow z)$

9) $x \rightarrow(y \vee z)=(x \rightarrow y) \vee(x \rightarrow z)$, for all $x, y, z \in L$.

Theorem 2.12: The direct product of Implicative Algebras are Implicative Algebra with point wise operation.

\section{Implichtive Almost Distributive Lattices} (IADLs)

In this section, we introduce the concept of Implicative Almost Distributive Lattice as a generalization of implicative algebra and study some properties and equivalent conditions of Implicative Almost Distributive Lattice.

Definition 3.1: Let $(L, \vee, \wedge, 0, m)$ be an ADL with 0 and maximal element $\mathrm{m}$. Then an algebra $\left(L, \vee, \wedge, \rightarrow,{ }^{\prime}, 0, m\right)$ of type $(2,2,2,1,0,0)$ is called Implicative Almost Distributive Lattice (IADL)if it satisfies the following conditions:
1) $x \vee y=(x \rightarrow y) \rightarrow y$

2) $x \wedge y=\left[(x \rightarrow y) \rightarrow x^{\prime}\right]^{\prime}$

3) $x \rightarrow(y \rightarrow z)=y \rightarrow(x \rightarrow z)$

4) $m \rightarrow x=x$

5) $x \rightarrow m=m$

6) $x \rightarrow y=y^{\prime} \rightarrow x^{\prime}$

7) $0^{\prime}=m$, for all $x, y, z \in L$.

Here after the symbol $\mathrm{L}$ stands an $\operatorname{IADL}\left(L, \vee, \wedge, \rightarrow{ }^{\prime}, 0, m\right)$ unless otherwise specified.

In the following lemma we give important properties of IADL.

Example 3.2: Let L be a discrete ADL with zero and with atleast two elements. Fix $m(\neq 0) \in L$ and define the binary operation $\rightarrow$ on $\mathrm{L}$ as follows, for any $x, y, \in L$,

$$
x \rightarrow y= \begin{cases}0 & \text { if } x \neq 0, \mathrm{y}=0 \\ m & \text { otherwise }\end{cases}
$$

and ' unary operation on L. Then clearly $\left(L, \vee, \wedge, \rightarrow,,^{\prime}, 0, m\right)$ is an implicative Almost distributive lattice and is called discrete IADL.

Example 3.3: Let $\mathrm{L}=\{0, x, y, z, m\}$ be a set with linear order $0<x<y<z<m$. Define the unary operation ' and the binary operation $\rightarrow$ on $\mathrm{L}$ as follows:

\begin{tabular}{|c|c|}
\hline $\mathrm{a}$ & $a^{\prime}$ \\
\hline 0 & $\mathrm{~m}$ \\
\hline $\mathrm{x}$ & $\mathrm{z}$ \\
\hline $\mathrm{y}$ & $\mathrm{y}$ \\
\hline $\mathrm{z}$ & $\mathrm{x}$ \\
\hline $\mathrm{m}$ & 0 \\
\hline
\end{tabular}

\begin{tabular}{|c|c|c|c|c|c|}
\hline$\rightarrow$ & 0 & $\mathrm{x}$ & $\mathrm{y}$ & $\mathrm{z}$ & $\mathrm{m}$ \\
\hline 0 & $\mathrm{~m}$ & $\mathrm{~m}$ & $\mathrm{~m}$ & $\mathrm{~m}$ & $\mathrm{~m}$ \\
\hline $\mathrm{x}$ & $\mathrm{z}$ & $\mathrm{m}$ & $\mathrm{m}$ & $\mathrm{m}$ & $\mathrm{m}$ \\
\hline $\mathrm{y}$ & $\mathrm{y}$ & $\mathrm{z}$ & $\mathrm{m}$ & $\mathrm{m}$ & $\mathrm{m}$ \\
\hline $\mathrm{z}$ & $\mathrm{x}$ & $\mathrm{y}$ & $\mathrm{z}$ & $\mathrm{m}$ & $\mathrm{m}$ \\
\hline $\mathrm{m}$ & 0 & $\mathrm{x}$ & $\mathrm{y}$ & $\mathrm{x}$ & $\mathrm{m}$ \\
\hline
\end{tabular}

Take $x \wedge y=\min \{x, y\}$ and $x \vee y=\max \{x, y\}$.

Then it is routine to verify $\left(L, \vee, \wedge, \rightarrow,{ }^{\prime}, 0, m\right)$ is an IADL. Lemma 3.4: In L, the following conditions hold and there proofs can easily be verified using Definition 3.1.

1) $[((x \rightarrow y) \rightarrow y] \wedge m=[(y \rightarrow x) \rightarrow x] \wedge m$

2) $\left[(x \rightarrow y) \rightarrow x^{\prime}\right]^{\prime} \wedge m=\left[\left((y \rightarrow x) \rightarrow y^{\prime}\right)^{\prime}\right] \wedge m$

3) $x \rightarrow x=m$

4) $m^{\prime}=0$

5) $\left(x^{\prime}\right)^{\prime}=x$ (is called involution )

6) $x^{\prime}=x \rightarrow 0$, for all $x, y \in L$.

The following theorem refers direct product of implicative almost distributive lattices and its proof is direct consequence of definition of IADL.

Theorem 3.5: Let $\mathrm{L}$ and $\mathrm{H}$ be two IADLs. Then $\mathrm{M}=L \times H$ is an IADL with point wise operation.

Example 3.6: Every implicative algebra $\left(L, \rightarrow,^{\prime}, 0,1\right)$ is an IADL.

In the following example we give a method of constructing an IADL which is neither IA nor discrete ADL.

Example 3.7: Let $\mathrm{L}$ be an implicative algebra and $\mathrm{D}$ be discrete IADL with 0 and at least two elements. Then $\mathrm{M}=$ $L \times D$ is an IADL with respect to point wise operation. But $\mathrm{M}$ is not Implicative algebra since $\mathrm{D}$ is not. Also $\mathrm{M}$ is not discrete IADL since $\mathrm{L}$ is not.

Now we define the following ideas in L: 
a. The relation $\leq$ on $\mathrm{L}$ is defined as for any $x, y \in L, x \leq y$ if and only if $x \rightarrow y=m$.

b. The maximal element $\mathrm{m}$ of $\mathrm{L}$ is defined as if $m \leq x$ for any $x \in L$,then $\mathrm{m}=\mathrm{x}$.

c. The principal ideal generated by the maximal element $\mathrm{m}$ of $\mathrm{L}$ is denoted $(\mathrm{m}]$ and defined as $(m]=\{m \wedge x: x \in L\}$.

Accordingly we have the following results:

1. The relation $\leq$ on $\mathrm{L}$ is a partial ordering.Thus $(L, \leq)$ is a poset.

2. For any $x, y \in L, x \wedge y=x \Leftrightarrow x \leq y$.

Now we have some results obtained in IADL,

Theorem 3.8: In L, the following conditions hold:

1) $0 \rightarrow x=m$

2) $x \vee m=m$

3) $x \wedge m=x$

4) $(\mathrm{m}]=\mathrm{L}$

5) $x \wedge y \leq x \rightarrow y$

6) $y \leq x \rightarrow y$

7) $x \wedge z \leq y$ implies $z \leq x \rightarrow y$

8) $x \leq y$ if and only if $z \rightarrow x \leq z \rightarrow y$ and $y \rightarrow z \leq x \rightarrow z$

9) $((x \rightarrow y) \rightarrow y) \rightarrow y=x \rightarrow y$

10) $x \rightarrow y \leq(y \rightarrow z) \rightarrow(x \rightarrow z)$

11) $(x \rightarrow z) \rightarrow(x \rightarrow y)=(z \rightarrow x) \rightarrow(z \rightarrow y)$.

12) $(x \vee y)^{\prime}=x^{\prime} \wedge y^{\prime}$

13) $(x \wedge y)^{\prime}=x^{\prime} \vee y^{\prime}$

14) $x \leq y, x \leq z$ implies $x \leq y \wedge z$

15) $y \leq x, z \leq x$ implies $(y \vee z) \leq x$

16) $(x \vee y) \rightarrow z \leq x \rightarrow z$ and $(x \vee y) \rightarrow z \leq y \rightarrow z$

17) $x \rightarrow z \leq(x \wedge y) \rightarrow z$ and $y \rightarrow z \leq(x \wedge y) \rightarrow z$

18) $(x \vee y) \rightarrow z=(x \rightarrow z) \wedge(y \rightarrow z)$

19) $(x \wedge y) \rightarrow z=(x \rightarrow z) \vee(y \rightarrow z)$

20) $x \rightarrow(y \wedge z)=(x \rightarrow y) \wedge(x \rightarrow z)$

21) $x \rightarrow(y \vee z)=(x \rightarrow y) \vee(x \rightarrow z)$, for all $x, y, z \in L$.

Remark 3.9: By 8 of Theorem 3.8, $x \leq y$, implies $y^{\prime} \leq x^{\prime}$ for any $x, y \in L$. Such condition is called order reversing.

Theorem 3.10: In L, the following conditions are equivalent for all $x, y \in L$,

1) $\mathrm{L}$ is Implicative Algebra

2) $x \wedge y \leq x, y \leq x \vee y$

3) $x \vee y$ is the least upper bound of $\{x, y\}$

4) $x \wedge y$ is the greatest lower bound for $\{x, y\}$

Proof: Let $x, y, z, t \in L .(1) \Rightarrow(2)$. Assume $\mathrm{L}$ is Implicative Algebra. Then $(x \wedge y) \rightarrow x=\left[(x \rightarrow y) \rightarrow x^{\prime}\right]^{\prime} \rightarrow x=(x \rightarrow y) \rightarrow$ $\left(x^{\prime} \rightarrow x^{\prime}\right)=(x \rightarrow y) \rightarrow 1=1$. Therefore, $x \wedge y \leq x$. Similarly, $x \wedge y \rightarrow y=1$ implies $x \wedge y \leq y$. And $x \rightarrow(x \vee y)=(x \rightarrow y) \rightarrow$ $(x \rightarrow y)=1$. Therefore, $x \leq x \vee y$. Similarly, $y \rightarrow(x \vee y)=1$. Therefore, $y \leq x \vee y$.

$(2) \Rightarrow(3)$. Assume (2).From 2, $x \vee y$ is an upper bound of $\{x, y\}$.Let $t$ be any other upper bound of $\{x, y\}$.Then $x \leq t$ and $y \leq t$.By Theorem 3.8 and our assumption, $x \vee y \rightarrow t=m$. Thus $x \vee y \leq t$. Hence, $x \vee y$ is least upper bound of $\{x, y\}$

$(3) \Rightarrow(2)$. Since $x \vee y$ is least upper bound of $\{x, y\}$, we have $x, y \leq x \vee y$ and $x \wedge(x \vee y)=x, y \wedge(x \vee y)=y$. Now $x \wedge$ $y=x \wedge(y \wedge(x \vee y))=y \wedge x \wedge(x \vee y)=y \wedge x$. This implies $\wedge$ is commutative and hence by Theorem $2.4 \mathrm{~L}$ is distributive lattice so that $x \wedge y \leq x, y$.
$(2) \Rightarrow(4)$. Assume (2) From 2, $x \wedge y$ is lower bound of $\{x, y\}$. Let $\mathrm{t}$ be any other lower bound of $\{x, y\}$. Then $t \rightarrow$ $x=m$ and $t \rightarrow y=m$. From Definition 3.1, Lemma 3.4, and Theorem 3.8, it follows that $t \rightarrow(x \wedge y)=m$. This implies $t \leq x \wedge y$.Hence, $x \wedge y$ is the greatest lower bound of $\{x, y\}$.

$(4) \Rightarrow(1)$. Assume 4 holds. From 4 , it follows $x \wedge y \leq x$ and $x \wedge y \leq y$. We know that $(L, \leq)$ is a poset. And also $x \wedge y \leq$ $x$ implies $x \wedge y=(x \wedge y) \wedge x=y \wedge x$. By Theorem $2.4, \mathrm{~L}$ is distributive lattice. Hence, $\mathrm{L}$ is an implicative algebra.

An IADL becomes an Implicative Algebra once it becomes a lattice. Therefore we give equivalent conditions for an IADL to become an Implicative Algebra.

Theorem 3.11: In L, the following are equivalent.

1) $\mathrm{L}$ is Implicative Algebra

2) The poset $(L, \leq)$ is directed above

3) $(L, \vee, \wedge)$ is a distributive lattice

4) $\vee$ is commutative

5) $\wedge$ is commutative

6) $\vee$ is right distributive over $\wedge$

7) The relation $\theta:=\{(x, y) \in L \times L: y \wedge x=x\}$ is anti symmetric.

Proof: $(1) \Rightarrow(2)$ : Assume $\mathrm{L}$ is an Implicative Algebra. Then $\mathrm{L}$ is lattice and for all $x, y \in L$ there exists $1 \in L$ such that $x \leq 1$ and $y \leq 1$. This implies the poset $(L, \leq)$ is directed above. $(2) \Rightarrow(3)$ : Assume the poset $(L, \leq)$ is directed above.Then from condition 10 of Theorem $2.3, \vee$ and $\wedge$ are commutative. This implies $(L, \vee, \wedge)$ is distributive lattice. (3) $\Rightarrow(4),(4) \Rightarrow$ (5) and $(5) \Leftrightarrow(6)$ are clear from Theorem 2.4. and $(5) \Leftrightarrow((7)$ is also clear.we finish the theorem by establishing $(7) \Rightarrow(1)$ : Assume (7). Then $(x \wedge y) \wedge(y \wedge x)=y \wedge x$ and $(y \wedge x) \wedge(x \wedge y)=$ $x \wedge y$ for any $x, y \in L$.so the elements $(x \wedge y, y \wedge x),(y \wedge x, x \wedge y)$ belongs to $\theta$, and hence $x \wedge y=y \wedge x$. In addition, by Theorem 3.10 , the poset $(L, \leq)$ has inf and sup so that $\mathrm{L}$ is a lattice. Therefore $\mathrm{L}$ is Implicative Algebra.

The following is also a characterization of an IADL. If $\mathrm{L}$ is an ADL with 0 , then for any $a \in L$, the interval $[0, \mathrm{a}]$ is a bounded distributive lattice [4]. Hence we can extend many concepts existing in the class of distributive lattices to the class of ADLs. The following theorem justifies the definition of IADL given in Definition 3.1.

Theorem 3.12: Let $\mathrm{L}$ be an ADL with 0 and a maximal element $\mathrm{m}$. Then the following are equivalent.

1) $\mathrm{L}$ is IADL

2) $[0, a]$ is Implicative Algebra for all $a \in L$

3) $[0, m]$ is Implicative Algebra.

Proof: $(1) \Rightarrow(2)$. Assume that L is an IADL and $a \in L$.we know that $[0, a]$ is a bounded distributive lattice.

Now, define a binary operation $\rightarrow^{a}$ on $[0, a]$ by $x \rightarrow^{a} y=$ $(x \rightarrow y) \wedge a$ for any $x, y \in[0, a]$.Let $x, y, z \in[0, a]$.

1) Since $x=x \wedge a$,and $y \wedge a=y$, we have, $x \rightarrow^{a}\left(y \rightarrow^{a} z\right)=$ $\left[x \rightarrow\left(y \rightarrow^{a} z\right)\right] \wedge a=[x \rightarrow((y \rightarrow z) \wedge a] \wedge a$ $=[x \rightarrow(y \rightarrow z)] \wedge a=[y \rightarrow(x \rightarrow z)] \wedge a=\left(y \rightarrow^{a}\left(x \rightarrow^{a} z\right)\right.$

2) $m \rightarrow^{a} x=(m \rightarrow x) \wedge a=x \wedge a=x$.

3) $x \rightarrow{ }^{a} m=(x \rightarrow m) \wedge a=m \wedge a=a=m$

4) $x \rightarrow^{a} y=(x \rightarrow y) \wedge a=\left(y^{\prime} \rightarrow x^{\prime}\right) \wedge a=y^{\prime} \rightarrow^{a} x^{\prime}$.

5) Prove similar to 1 .

6) $0^{\prime}=0 \rightarrow^{a} 0=(0 \rightarrow 0) \wedge m=m \wedge m=m=a$ 
Therefore, [0, a] is an Implicative Algebra for all $a \in L$.

$(2) \Rightarrow(3)$. It is trivial.

$(3) \Rightarrow(1)$. Assume that $[0, m]$ is an Implicative Algebra in which the binary operation is $(\rightarrow)$ denoted by $\rightarrow^{m}$. Define $x \rightarrow y=x \wedge m \rightarrow^{m} y \wedge m$ for any $x, y \in L$. Let $x, y, z \in L$.

1) $x \vee y=\left[x \wedge m \rightarrow^{m} y \wedge m\right] \wedge m \rightarrow^{m} y \wedge m=(x \rightarrow y) \rightarrow y$

2) $x \wedge y=\left[\left(x \wedge m \rightarrow^{m} y \wedge m\right) \wedge m \rightarrow^{m} x^{\prime} \wedge m\right]^{\prime}=[(x \rightarrow y) \rightarrow$ $\left.x^{\prime}\right]^{\prime}$

3) $x \rightarrow(y \rightarrow z)=x \wedge m \rightarrow^{m}\left(y \wedge m \rightarrow^{m} z \wedge m\right) \wedge m=y \wedge$ $m \rightarrow^{m}\left(x \wedge m \rightarrow^{m} z \wedge m\right)=y \wedge m \rightarrow^{m}\left(x \wedge m \rightarrow^{m} z \wedge m\right) \wedge$ $m=y \rightarrow(x \rightarrow z)$

4) $\left.x \rightarrow y=x \wedge m \rightarrow^{m} y \wedge m=(y \wedge m)^{\prime} \rightarrow^{m}(x \wedge m)^{\prime}\right)=y \prime \rightarrow x \prime$

5) $m \rightarrow x=m \wedge m \rightarrow^{m} x \wedge m=x$

6) $x \rightarrow m=x \wedge m \rightarrow^{m} m \wedge m=m$

7) $0^{\prime}=0 \rightarrow 0=0 \wedge m \rightarrow^{m} 0 \wedge m=m$

Therefore, $\mathrm{L}$ is IADL.

Lemma 3.13: Let $x, y \in L$. Then the following are equivalent.

1) $(x] \subseteq(y]$

2) $y \wedge x=x$

3) $x \wedge t \leq y \wedge t$ all $t \in L$

Proof: We can easily prove using Lemma 3.4 and Theorem 3.8.

Theorem 3.14: . Let $\mathrm{L}$ be an ADL with maximal element $\mathrm{m}$. then $\mathrm{L}$ is an IADL if and only if the set of principal ideal $\mathrm{PI}(\mathrm{L})$ is an implicative algebra.

Proof: Let $\mathrm{L}$ be an ADL with maximal element $\mathrm{m}$. Suppose $\mathrm{L}$ is an IADL, then since $(0]$ and $(\mathrm{m}]$ are least and greatest element of $\mathrm{PI}(\mathrm{L}),(P I(L), \vee, \wedge)$ is bounded. Clearly $(P I(L), \subseteq)$ is a poset under set inclusion $\subseteq$. For $(x],(y] \in$ $P I(L),(x] \wedge(y]=(x \wedge y]=\inf \{(x],(y]\}$ and $(x] \vee(y]=(x \vee y]=$ $\sup \{(x],(y]\}$.Therefore, $(P I(L), \vee, \wedge)$ is bounded lattice. Now we define $(x] \rightarrow(y]=(x \rightarrow y]$ for any $x, y \in L$. Using Lemma 3.13 we prove that the binary operation $\rightarrow$ on $\mathrm{PI}(\mathrm{L})$ is well defined.If (a]=(b] and (c]=(d), then $a \wedge b=b, b \wedge a=a, c \wedge d=$ $d, d \wedge c=c$. Now $a \rightarrow c=a \rightarrow(d \wedge c)=(a \rightarrow d) \wedge(a \rightarrow c)=$ $((a \vee b) \rightarrow d) \wedge(a \rightarrow c)=(a \rightarrow d) \wedge(b \rightarrow d) \wedge(a \rightarrow c) \leq(b \rightarrow$ $d) \wedge(a \rightarrow c) \leq(a \rightarrow c$. Hence $(b \rightarrow d) \wedge(a \rightarrow c)=(a \rightarrow c)$. This implies that $(a \rightarrow c] \subseteq(b \rightarrow d]$ and similarly we get $(b \rightarrow d] \subseteq(a \rightarrow c]$. Therefore $(a \rightarrow c)=(b \rightarrow d]$.Thus the binary operation $\rightarrow$ on $\mathrm{PI}(\mathrm{L})$ is well defined.Now we can routinely verify that $\mathrm{PI}(\mathrm{L})$ is an implicative algebra. Conversely, let $\mathrm{L}$ be an ADL with maximal element $m$. Suppose $((P I(L), \vee, \wedge)$ is an implicative algebra.For all $x, y \in L$, define $x \rightarrow y=z \wedge m$ where $(x] \rightarrow(y]=(z]$ for some $z \in L$. Let (s]=(t], for some $s, t \in L$. Then $s \wedge t=t$ and $t \wedge s=s$. Now $s \wedge m=t \wedge s \wedge m=s \wedge t \wedge m=t \wedge m$. Thus the binary operation $\rightarrow$ on $\mathrm{L}$ is well defined. Let $x, y, z \in L$.

1) $(x] \vee(y]=((x] \rightarrow(y]) \rightarrow(y]=(r] \rightarrow(y]$ so that $x \rightarrow y=$ $r \wedge m$ for some $r \in L$ where $(x] \rightarrow(y]=(r]$ and $r \rightarrow y=$ $v \wedge m$ for some $v \in L$ where $(r] \rightarrow(y]=(v]$. Therefore $(x \rightarrow y) \rightarrow y=v \wedge m$ where $((x] \rightarrow(y]) \rightarrow(y]=(v]$.

2) $(x] \wedge(y]=\left(((x] \rightarrow(y]) \rightarrow\left(x^{\prime}\right]\right)^{\prime}=\left[(r] \rightarrow\left(x^{\prime}\right]^{\prime}\right.$ where $(x] \rightarrow(y]=(r]$ for some $r \in L$ and $\left((r] \rightarrow\left(x^{\prime}\right]\right)^{\prime}=(v]^{\prime}$ for some $v \in L$. Therefore $\left[(x \rightarrow y) \rightarrow x^{\prime}\right]^{\prime}=v^{\prime} \wedge m$

3) Let $(x],(y],(z] \in P I(L)$ and $\operatorname{PI}(\mathrm{L})$ is an implicative algebra. $(x] \rightarrow((y] \rightarrow(z])=(y] \rightarrow((x] \rightarrow(z])$ such that $x \rightarrow r=v \wedge m$ where $(x] \rightarrow(r]=(v]$ and $y \rightarrow z=r \wedge m$ where $(y] \rightarrow(z]=(r]$ for some $r, v \in L$. This implies $x \rightarrow(y \rightarrow z)=v \wedge m$ where $(y] \rightarrow((x] \rightarrow(z])=(x] \rightarrow$ $((y] \rightarrow(z])=(v]$ for some $v \in L$. This also implies $y \rightarrow(x \rightarrow z)=v \wedge m$ where $(y] \rightarrow((x] \rightarrow(z])=(v]$ for some $v \in L$. Therefore $x \rightarrow(y \rightarrow z)=y \rightarrow(x \rightarrow z)$.

4) $(x] \rightarrow(y]=(r]$ implies $x \rightarrow y=r \wedge m$ for some $r \in L$. Now $y^{\prime} \rightarrow x^{\prime}=(y \rightarrow 0) \rightarrow(x \rightarrow 0)=x \rightarrow((y \rightarrow 0) \rightarrow$ $0)=x \rightarrow y=r \wedge m$ where $\left(y^{\prime}\right] \rightarrow\left(x^{\prime}\right]=(r]$.Therefore $x \rightarrow y=y^{\prime} \rightarrow x^{\prime}$.

5) $(m] \rightarrow(x]=(x]$ implies $m \rightarrow x=x \wedge m=x$.

6) $(x] \rightarrow(m]=(m]$ implies $x \rightarrow m=m \wedge m=m$.

7) $\left(0^{\prime}\right]=(m]$ implies $0 \rightarrow 0=m \wedge m=m$. Therefore $\mathrm{L}$ is an IADL.

Remark 3.15: In $\mathrm{L}$ with $0 \neq m, \rightarrow$ can never be associative.

Proof: Suppose $\rightarrow$ on $\mathrm{L}$ is associative and $0 \neq m$. Let $a=b=c \in L$ in condition 3 of Definition 3.1. Then $(a \rightarrow$ $a) \rightarrow a=a \rightarrow(a \rightarrow a)$. This implies $m \rightarrow a=a \rightarrow m$ implies again $\mathrm{a}=\mathrm{m}$, Hence, $0=\mathrm{m}$, leads a contradiction. Therefore, $\rightarrow$ can never be associative.

\section{REFERENCES}

[1] X. Yang, "Lattice implication algebras [j]," Journal of Southwest Jiaotong University, vol. 1, 1993.

[2] Y. Xu and K. Qin, "implication algebras and lattice implication algebra classes, j," Hebei Mining and Civil Engineering Institute, vol. 3, pp. 139143, 1992.

[3] X. Yang, L. Jun, S. Zhenming, and Q. Keyun, "On semantics of 1-valued first-order logic lvft," International Journal of General System, vol. 29, no. 1 , pp. 53-79, 2000.

[4] V. Kolluru and B. Bekele, "Implicative algebras," Momona Ethiopian Journal of Science, vol. 4, no. 1, pp. 90-101, 2012.

[5] U. Swamy and G. Rao, "Almost distributive lattices," Journal of the Australian Mathematical Society, vol. 31, no. 1, pp. 77-91, 1981. 